\title{
Editorial
}

Nephrology

\section{Uric Acid and Decline in Renal Function-Partners in Crime}

\author{
Jesse Dawson Patrick B. Mark \\ Institute of Cardiovascular and Medical Sciences, BHF Glasgow Clinical Research Centre, \\ University of Glasgow, Glasgow, UK
}

Uric acid is a breakdown product of purine metabolism, and its production is regulated by the enzyme xanthine oxidase (XO). Pre-clinical studies suggest that hyperuricemia induces hypertension, renal microvascular disease, and endothelial dysfunction, and that it causes triglyceride accumulation in response to fructose ingestion [1]. XO may have further, uric acid-independent, potentially harmful effects on the cardiovascular system. $\mathrm{XO}$ action generates superoxide, which has a role in renin-angiotensin system activation, detrimental responses to fructose ingestion, and ischemia-reperfusion injury.

In this paper, Kuwabara et al. [2] explored the relationship between serum uric acid level and change in estimated glomerular filtration rate (eGFR) over a 5 -year period in a single-center retrospective cohort study. This was a well-conducted analysis including over 13,000 Japanese patients. Higher baseline serum uric acid level was associated with greater odds of rapid eGFR decline (OR 1.27, 95\% CI 1.17-1.38 for each $1 \mathrm{mg} / \mathrm{dL}$ increment in serum uric acid). The authors were able to adjust for numerous potential confounders including age, body mass index, cardiovascular risk factors, and diabetes status but were not able to account for the difference in medication use or the presence of proteinuria. The analyses were stratified by gender, and the sub-analyses stratified by baseline eGFR found similar results. This association has been shown before in Japanese patients and in those treated for hypertension in the UK [3]. A novel finding from this study was that an increase in serum uric acid over a 5-year period was associated with a greater decline in eGFR (OR 3.77, 95\% CI 3.35-4.26 for each $1 \mathrm{mg} / \mathrm{dL}$ increase). Whilst, as the authors acknowledge, dissecting an independent effect of uric acid on decline in eGFR is challenging due to the effect of reducing kidney function on serum uric acid levels. Taken together, the epidemiological data are consistent, large in scale, and suggest serum uric acid is associated with development of cardiovascular, renal, and metabolic diseases. However, these data can only demonstrate association. Experimental data do support causality. Induction of hyperuricemia causes a decline in renal function, glomerular hypertension, and a small vessel renal arteriopathy in rodent models. This may mediate reno-vascular disease via increased renin production, COX-2 expression, and thromboxane production [1].

One method of trying to better assess causality in humans is via the use of Mendelian randomization studies. Here, the relationship between a genetic polymorphism known to affect the exposure of interest (in this case, uric acid level) and outcome is explored. This association should not be susceptible to confounding or reverse causation as the presence of the phenotype of interest will be randomly determined at conception. Previous Mendelian randomization studies have failed to demonstrate a clear association between instrumental variables for serum

\section{KARGER}

(c) 2017 S. Karger AG, Basel 
uric acid and chronic kidney disease (CKD), and in one study, the instrumental variable was associated with better renal function in men [4]. Mendelian randomization studies in this setting have numerous challenges. Several genes are associated with serum uric acid levels with renal urate transports accounting for most of the variance. Renal transport of serum uric acid is complex and it may be that activity of the transporters is related to renal function, rather than serum uric acid level itself. The mechanism of hyperuricemia and whether intracellular levels are raised may be important with regard to the risk conveyed and our understanding of how this affects reported epidemiological relationships, or how it is affected by genotype, is limited. It is worth noting that a number of genes associated with CKD in genome-wide association studies are also associated with serum uric acid level.

So, epidemiological and experimental data suggest that hyperuricemia may contribute to renal function decline but findings from Mendelian randomization studies leave this open to debate. The more important question for clinicians is whether uric acid reduction slows decline in renal function and whether this reduction is sufficient to translate into either a reduction in progression to endstage renal disease (ESRD), requiring dialysis or transplantation, or a reduction in the increased cardiovascular risk associated with CKD. Studies have reported an effect of allopurinol, the most widely used uric acid lowering drug, on renal function since the 1970s. It is important to recognize that $\mathrm{XO}$ inhibitors have clear pleotropic effects, perhaps mediated by reduced formation of reactive oxygen species. Serum uric acid level can also be lowered by uricosuric drugs, such as probenecid, which do not directly reduce oxidative stress. Thus, an observed effect of allopurinol cannot be assumed to be due to uric acid reduction.

Clinical trials suggest that allopurinol improves or delays decline in renal function. Although many of the early reports were from uncontrolled or open label studies, data from randomized and blinded trials are emerging. However, these studies have typically been small. Meta-analysis of these small studies ( 8 trials, $n=476$ ) showed only a difference in change in serum creatinine but not in eGFR [5]. There is as yet no evidence that allopurinol use will reduce the rate of progression to ESRD. This may be simply because it has not been adequately studied. There have been no adequately powered trials to confirm or refute an effect. There are also insufficient data to draw conclusions about the effect of probenecid in the setting of CKD, and whilst it may be an ineffective uricosuric in $\mathrm{CKD}$, trials testing allopurinol compared to allopurinol in patients with heart failure showed that allopurinol $600 \mathrm{mg}(n=30)$ and not probenecid $(n=26)$ improved forearm endothelial function [6] despite similar degrees of uric acid reduction. The cardiovascular effects of allopurinol, at least, may not be mediated via uric acid reduction. We do not know if this is the case with renal outcomes.

No large trials of cardiovascular outcomes exist in patients with CKD, although data support the need for these to be performed. In a randomized, double-blind, placebocontrolled, parallel-group study in patients with stage $3 \mathrm{CKD}$ and left ventricular hypertrophy (LVH), allopurinol $300 \mathrm{mg}$ daily reduced LVH and improved endothelial function over a 9-month treatment period [7]. LVH is an independent predictor of cardiovascular events in CKD and $\mathrm{LVH}$ regression appears beneficial. This raises the possibility that allopurinol will improve $\mathrm{CV}$ outcomes in patients with CKD. This notion is being tested in a double-blind, placebo-controlled trial of allopurinol in hemodialysis patients aiming to regress LVH (ClinicalTrials. gov NCT01951404).

The paper by Kuwabara et al. [2] is an important step forward. It adds fuel to fire surrounding the importance of uric acid in cardiovascular and renal disease - a fire that has burned for over half a century. We need to now conduct thoughtful prospective interventional studies of uric acid reduction. Large-scale trials are needed to assess the effect of XO inhibition on meaningful clinical outcomes in patients with CKD, such as progression to ESRD or cardiovascular outcomes. XO inhibitors have the most data to support conducting of large-scale trials but smaller trials, perhaps with change in eGFR as an end point, are ideally needed to compare the effects of XO inhibition and uricosuric drugs. This will establish whether effects in renal disease are uric acid mediated or due to other pleotropic effects.

\section{Disclosure Statement}

J. Dawson holds substantial charitable research funding to explore allopurinol use after stroke.

References

1 Cirillo P, Sato W, Reungjui S, et al: Uric acid, the metabolic syndrome, and renal disease. J Am Soc Nephrol 2006;17(12 suppl 3):S165S168.

2 Kuwabara M, Bjornstad P, Hisatome I, et al: Elevated serum uric acid level predicts rapid decline in kidney function. Am J Nephrol 2017;45:330-337. 
3 Dawson J, Jeemon P, Hetherington L, et al: Serum uric acid level, longitudinal blood pressure, renal function, and long-term mortality in treated hypertensive patients. Hypertension 2013;62:105-111.

4 Hughes K, Flynn T, de Zoysa J, Dalbeth N, Merriman TR: Mendelian randomization analysis associates increased serum urate, due to genetic variation in uric acid transporters, with improved renal function. Kidney Int 2014;85:344-351.
5 Bose B, Badve SV, Hiremath SS, et al: Effects of uric acid-lowering therapy on renal outcomes: a systematic review and meta-analysis. Nephrol Dial Transplant 2014;29:406413.
6 George J, Carr E, Davies J, Belch JJ, Struthers A: High-dose allopurinol improves endothelial function by profoundly reducing vascular oxidative stress and not by lowering uric acid. Circulation 2006;114:25082516.

7 Kao MP, Ang DS, Gandy SJ, Nadir MA, Houston JG, Lang CC, et al: Allopurinol benefits left ventricular mass and endothelial dysfunction in chronic kidney disease. J Am Soc Nephrol 2011;22:1382-1389. 